\title{
Validação de metodologias analíticas para determinação quantitativa de $\alpha$-tocoferol e 4-nerolidilcatecol
}

\author{
Cristina Dislich Ropke*, Elissa Arantes Ostrosky, Telma Mary Kaneko, César Moisés Camilo, \\ Tânia Cristina Higashi Sawada, Silvia Berlanga de Moraes Barros
}

Departamento de Análises Clínicas e Toxicológicas, Faculdade de Ciências Farmacêuticas, Universidade de São Paulo

*Correspondência:

C. D. Ropke

Departamento de Análises Clínicas e

Toxicológicas

Faculdade de Ciências Farmacêuticas

Universidade de São Paulo

Av. Lineu Prestes, 580

05508-900 - São Paulo - SP

Brasil

E-mail: cdropke@usp.br

\begin{abstract}
O objetivo do nosso trabalho foi desenvolver um método para avaliação da concentração do $\alpha$-tocoferol, considerado o antioxidante lipofilico de maior importância, e do 4-nerolidilcatecol (4-NC), uma substância natural com comprovada ação antioxidante in vitro $e$ in vivo, em matriz biológica (homogeneizado de pele). Utilizamos a cromatografia de alta eficiência acoplada a um detector eletroquímico, sendo que o método apresentou linearidade para as concentrações de $0,025 \mu \mathrm{g} / \mathrm{mL}$ a $0,1 \mu \mathrm{g} / \mathrm{mL}$ para o $\alpha-T$ (tempo de retenção 3, $4 \mathrm{~min}$ ) e de 0,15 $\mu \mathrm{g} / \mathrm{mL}$ a 2,5 $\mu \mathrm{g} / \mathrm{mL}$ para o 4-NC (tempo de retenção 2,06 $\mathrm{min}$ ), dissolvidos em etanol e etanol:água (1:1). A taxa de recuperação do $\alpha$-T adicionado nas concentrações de 0,5; 0,1 e 0,025 $\mathrm{g} / \mathrm{mL}$ aos homogeneizados de pele foi de 94,03; 111,2 e 80,7\%, respectivamente. A taxa de recuperação de 4-NC adicionado nas concentrações de 2,5; 0,625 e 0,156 $\mu \mathrm{g} / \mathrm{mL}$ foi de 103,7; 91,7 e 91,7\%. Este método analítico foi e está sendo empregado, com sucesso devido à sua precisão e rapidez, em diversas análises do laboratório.
\end{abstract}

Unitermos:

- Pothomorphe umbellata

- 4-Nerolidilcatecol

- CLAE

- Validação

\section{INTRODUÇÃO}

A pele é o maior orgão do nosso corpo e encontrase constantemente exposta às fontes geradoras de radicais livres, como a radiação ultravioleta, poluentes do ar e radiação ionizante. O dano causado por radicais livres e espécies reativas de oxigênio pode ter como conseqüencia o fotoenvelhecimento e o câncer de pele (Black, 1987; Juerkivicz, Buetner, 1994). Neste contexto, a aplicação tópica de antioxidantes vem sendo considerada estratégia promissora na prevenção do dano oxidativo à pele. Resultados de estudos pré-clínicos e clínicos consideráveis in- dicam o uso potencial de $\alpha$-tocoferol, na prevenção do dano fotooxidativo (Bisset, 1990; Lopez-Torres et al, 1997; Fuchs, 1998; Mayer; 1993). Além disto, a depleção de $\alpha$-tocoferol na pele foi considerado um marcador "in vivo" de estresse oxidativo cutâneo induzido por radiação ultravioleta (Thiele et al., 1998). Outras substâncias de origem vegetal, como flavonóides e outros compostos fenólicos, têm sido propostos, para aplicação tópica com objetivo de prevenir o dano fotooxidativo à pele (Bonina et al., 1996; Saija et al., 2000). Um estudo de avaliação antioxidante na pele de camundongos Hairless demonstrou que a aplicação tópica do extrato liofilizado de raízes 
de Pothomorphe umbellata foi capaz de causar redução de $97 \%$ nos indicadores de lipoperoxidação, como produção de substância reativa ao ácido tiobarbitúrico e a emissão de quimiluminescência. Esta atividade antioxidante foi cerca de 2,5 vezes maior que a do $\alpha$-tocoferol aplicado nas mesmas condições, sugerindo o emprego desse extrato em formulações cosméticas, que têm por objetivo combater os efeitos danosos causados por radicais livres (Ropke, 2000). A atividade antioxidante do extrato liofilizado da raiz de Pothomorphe umbellata, quando avaliada em ensaios in vitro, foi, em parte, atribuída à presença do 4nerolidilcatecol (4-NC) (Barros et al., 1996), um composto fenólico isolado das folhas e raízes deste vegetal (Kijjoa, 1980).

É crescente o emprego de métodos cromatográficos na análise quantitativa de substâncias de interesse biomédico. Para que estes métodos tenham utilidade é necessário que os dados gerados, principalmente aqueles obtidos a partir de matrizes biológicas, sejam confiáveis (Causon, 1997). Conforme definição da UNITED STATES PHARMACOPEIA (1995), a validação de métodos assegura a credibilidade destes durante o uso rotineiro, sendo algumas vezes mencionado como "o processo que fornece uma evidência documentada de que o método realiza aquilo para o qual é indicado". Constada a relevância da atividade antioxidante do 4-NC tornou-se imprescindível o desenvolvimento de metodologia analítica para a sua aplicação em estudos de permeação desta substância a partir de diferentes formulações, e em estudos de atividade antioxidante in vivo. Considerando a importância do $\alpha$-tocoferol como antioxidante na pele julgamos pertinente validar metodologia para determinação desta substância possibilitando o acompanhamento de sua concentração nos estudos in vivo. O objetivo do nosso trabalho foi então desenvolver um método validado para quantificação de $\alpha$-tocoferol e 4-nerolidilcatecol em pele, baseando-se, para isto, no "GUIA PARA VALIDAÇÃO DE MÉTODOS ANALÍTICOS" publicado pela ANVISA através da resolução-RE n 475, de 19 de março de 2002.

\section{MATERIAL E MÉTODOS}

\section{Reagentes}

Metanol, etanol e hexano Lichrosolv ${ }^{\circledR}$ e $\mathrm{KCl}$ foram de procedência Merck (Darmstadt, Alemanha). $\mathrm{LiClO}_{4}$ foi obtido da Aldrich. O 4-nerolidilcatecol, utilizado como substância química de referência, foi uma doação do Prof. Massuo Jorge Kato do Instituto de Química da USP (estrutura confirmada por RMN) e o $\alpha$-tocoferol utilizado como padrão foi adquirido da SIGMA.

\section{Validação da metodologia analítica}

$\mathrm{Na}$ validação foram seguidas as orientações do "Guia para validação de métodos analíticos" (ANVISA, 2002).

- A curva de calibração foi construída a partir das sextuplicatas de 5 concentrações diferentes, sendo o cálculo de regressão linear realizado pelo método dos mínimos quadrados.

- Precisão (intra e inter-corrida) foi expressa como coeficiente de variação $(\mathrm{CV} \%)$, segundo a fórmula: $\mathrm{CV}=$ $\mathrm{DP} / \mathrm{CMD}$, em que DP é o desvio padrão e CMD, a concentração média determinada.

- Exatidão (inter e intra-dia) foi expressa pela relação entre a concentração média determinada experimentalmente e a concentração teórica correspondente.

- Limite de detecção (LD) foi expresso pela equação: $\mathrm{LD}=(\mathrm{DP} X 3,3) / \mathrm{ic}$, em que DP é o desvio padrão do intercepto com o eixo Y de três curvas de calibração e ic é a inclinação da curva de calibração.

- Limite de quantificação (LQ) foi expresso pela equação: $L Q=(D P X 10) / i c$.

- Avaliação da estabilidade do 4-NC em solução etanol: água $(1: 1)$ a $37^{\circ} \mathrm{C}$ em cinco tempos diferentes $(0 ; 8$; $10 ;, 11,5$ e $24 \mathrm{~h})$

- Curvas de recuperação para o $\alpha$-tocoferol e o 4-NC foram construídas a partir dos homogeneizados de pele. Os princípios ativos foram adicionados ao tubo de homogeneização, antes desse processo, levando em consideração as diluições do processo de extração de modo que ao final da extração tivéssemos as concentrações esperadas. Para os ensaios de recuperação utilizamos uma adaptação do método proposto por Burton (Burton \& Ingold, 1985) para extração de atocoferol de tecidos. Homogeneizaram-se100 mg de tecido (pele) em um homogeneizador Ultra-Turax, em $1 \mathrm{~mL}$ de tampão fosfato de sódio $0,1 \mathrm{M} \mathrm{pH} \mathrm{7,0.} \mathrm{Adicio-}$ naram-se $300 \mu \mathrm{L}$ de dodecil sulfato de sódio (SDS) e $600 \mu \mathrm{L}$ de metanol a $300 \mu \mathrm{L}$ deste homogeneizado e agitou-se em vórtex durante $20 \mathrm{~s}$. Ao tubo foram, então, adicionados $6 \mathrm{~mL}$ de hexano e a mistura foi agitada no vórtex durante 3 minutos. Após breve centrifugação (3 min,1000 rpm) a fração hexânica foi retirada $(3 \mathrm{~mL})$ e evaporada em atmosfera de nitrogênio. As amostras foram então ressuspendidas em $500 \mu \mathrm{L}$ de etanol Lichrosolv. Para construção da curva de recuperação de 4-NC foram adicionadas ao homogeneizado de pele as concentrações de $2,5 \mu \mathrm{g} / \mathrm{mL} ; 0,625 \mu \mathrm{g} / \mathrm{mL}$; $0,156 \mu \mathrm{g} / \mathrm{mL}$. Para construção da curva de recuperação de $\alpha$-tocoferol foram escolhidas as concentrações de $0,5 \mu \mathrm{g} / \mathrm{mL} ; 0,1 \mu \mathrm{g} / \mathrm{mL} ; 0,025 \mu \mathrm{g} / \mathrm{mL}$. 


\section{Sistema cromatográfico e condições do detector}

O sistema cromatográfico foi composto de bomba Waters Model 510, injetor 7161 Rheodyne dotado de loop de $20 \mu \mathrm{L}$, detector eletroquímico HP1049A, constituído de um eletrodo de trabalho de placas de carbono-vítreo e um eletrodo de referência de estado sólido. O sinal produzido foi transmitido a um integrador SP4600 Termoseparation Products. A fase móvel utilizada foi metanol:água 90:10, contendo $\mathrm{KCl} 2 \mathrm{mM} \mathrm{e} \mathrm{LiClO}_{4} 20 \mathrm{mM}$ para o 4-NC e metanol:água 95:5, contendo $\mathrm{KCl} 2 \mathrm{mM}$ e $\mathrm{LiClO}_{4} 20 \mathrm{mM}$ para o $\alpha$-tocoferol, com um fluxo $1.0 \mu \mathrm{L} / \mathrm{min}$. Coluna Supelcosil LC-8, $3 \mu \mathrm{m}, 75$ X 4,6 mm (Supelco, Bellefonte, PA, USA). O detector eletroquímico foi programado da seguinte forma: potencial $=0,600 \mathrm{~V}$; polaridade $=$ oxidação $;$ modo $=$ amperometria.

\section{RESULTADOS}

\section{Linearidade do método analítico para análise de 4-NC}

A completa dissolução do princípio ativo 4-NC foi obtida empregando-se a solução etanol: água (1:1). O tempo de retenção do 4-NC no sistema cromatográfico proposto foi de 2,14 minutos. A Figura 1 mostra a relação linear entre as concentrações de 4-NC e as áreas dos picos, tendo sido obtido o coeficiente de correlação $\left(\mathrm{R}^{2}\right)$ de 0,998 .

A partir das fórmulas descritas anteriormente calculamos o limite de detecção de $0,05 \mu \mathrm{g} / \mathrm{mL}$ e o limite de quantificação de $0,1767 \mu \mathrm{g} / \mathrm{mL}$ para o $4-\mathrm{NC}$.

TABELA I - Dados da curva de calibração de 4-NC dissolvido em etanol:água (1:1)

\begin{tabular}{rcccccc}
\hline $\begin{array}{c}\text { Concentração } \\
\mu \mathrm{g} / \mathrm{mL}\end{array}$ & $\begin{array}{c}\text { Média da área } \\
\text { do pico* }\end{array}$ & $\begin{array}{c}\text { Desvio } \\
\text { Padrão }\end{array}$ & $\begin{array}{c}\text { CV\% inter- } \\
\text { corrida }\end{array}$ & $\begin{array}{c}\text { CV\% intra- } \\
\text { corrida }\end{array}$ & $\begin{array}{c}\text { Exatidão } \\
\text { inter-dia } \\
\text { (três dias } \\
\text { consecutivos) }\end{array}$ & $\begin{array}{c}\text { Exatidão } \\
\text { intra-dia }\end{array}$ \\
\hline 2,5 & 795876 & 58312 & 7,3 & 2,4 & 103,32 & 99,98 \\
1,25 & 392632 & 17989 & & & & 106,10 \\
0,625 & 202265 & 28458 & 14 & 3,5 & 106,34 & 10 \\
0,313 & 94598 & 13126 & & & & 93,11 \\
0,156 & 38911 & 10727 & 27 & 18 & 91,61 \\
\hline
\end{tabular}

*média de no mínimo 6 injeções; CV - coeficiente de variação

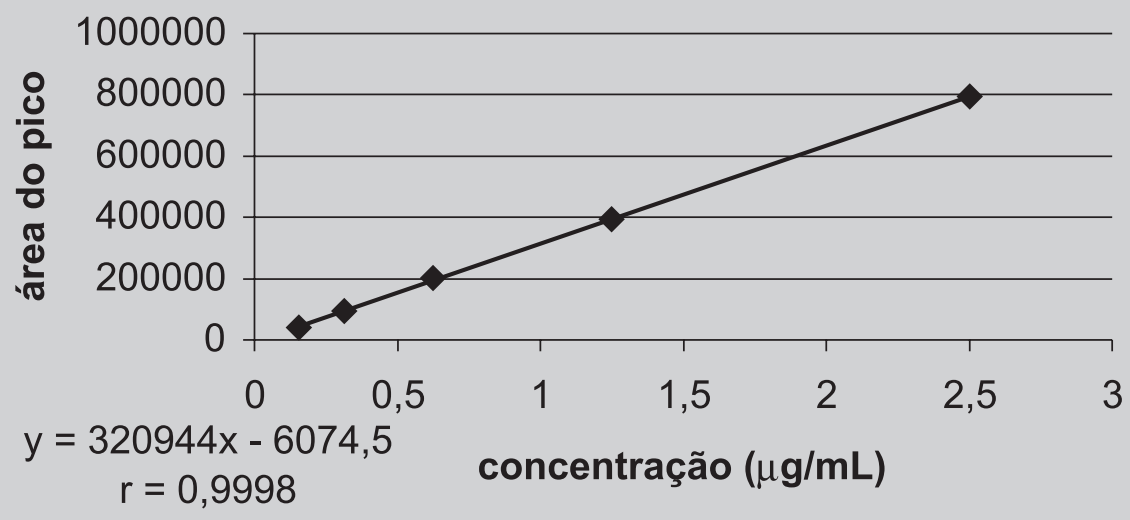

FIGURA 1 - Curva de calibração para análise do 4 -NC no intervalo de concentrações de 0,156 a $2,5 \mu \mathrm{g} / \mathrm{mL}$ de 4 -NC dissolvido em etanol:água referente à linearidade do método. 


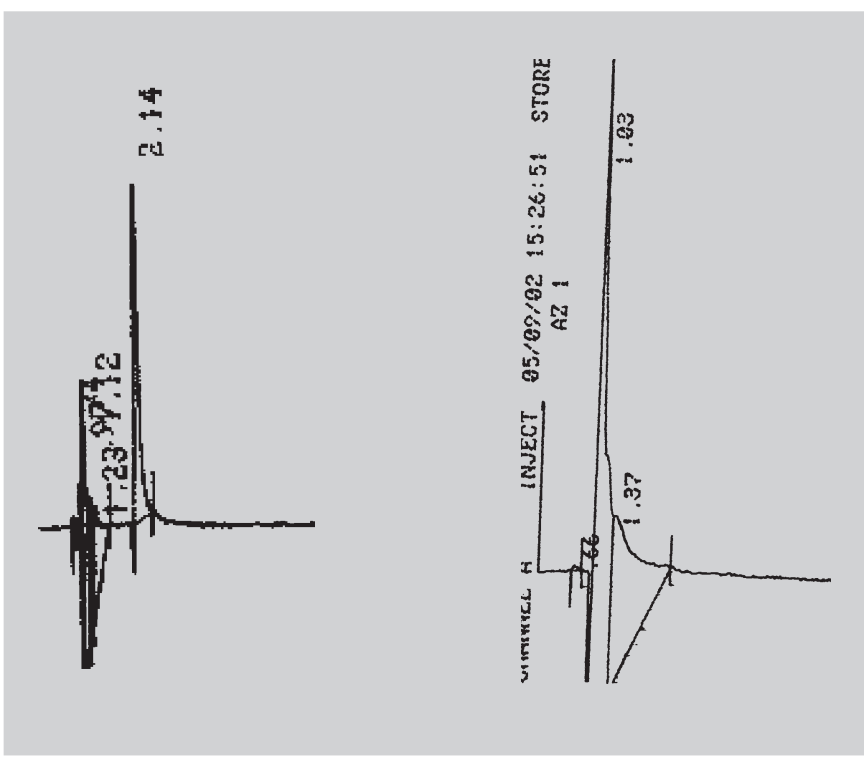

FIGURA 2 - (A) Cromatograma do padrão de 4-NC em ETOH: $\mathrm{H}_{2} \mathrm{O}$, na concentração de $0,625 \mu \mathrm{g} / \mathrm{mL}$ (tempo de retenção 2,14), (B) cromatograma da extração do homogeneizado de pele.
A curva de calibração do 4-NC dissolvido em etanol: água foi feita para analisar as amostras retiradas do compartimento receptor da célula de Franz. Enquanto que o etanol foi o solvente empregado para ressuspender o princípio ativoapós o processo de extração. A Figura 3 mostra a curva de calibração do 4-NC, no intervalo de concentração de 0,156 a $2,5 \mu \mathrm{g} / \mathrm{mL}$, dissolvido em etanol. o coeficiente de correlação encontrado foi de 0,998 indicando a linearidade do método analítico. A partir das fórmulas descritas anteriormente, calculamos o limite de detecção de $0,057 \mu \mathrm{g} / \mathrm{mL}$ e o limite de quantificação de $0,175 \mu \mathrm{g} / \mathrm{mL}$ para o $4-\mathrm{NC}$ dissolvido em etanol.

\section{Recuperação de 4-NC em homogeneizado de pele}

O 4-NC nas concentrações de 0,$156 ; 0,625$ e $2,5 \mu \mathrm{g} / \mathrm{mL}$ foi adicionado ao homogeneizado de pele (antes da homogeneização) e submetido ao processo de extração.A tabela III mostra as porcentagens de recuperação para as diferentes concentraçções de 4-NC adicionado aos homogeneizados.

TABELA II - Dados da curva de calibração de 4-NC, dissolvido em etanol Lichrosolv

\begin{tabular}{rcccccc}
\hline $\begin{array}{c}\text { Concentração } \\
\mu \mathrm{g} / \mathrm{mL}\end{array}$ & $\begin{array}{c}\text { Média da área } \\
\text { do pico* }\end{array}$ & $\begin{array}{c}\text { Desvio } \\
\text { Padrão }\end{array}$ & $\begin{array}{c}\text { CV\% inter- } \\
\text { corrida }\end{array}$ & $\begin{array}{c}\text { CV\% intra- } \\
\text { corrida }\end{array}$ & $\begin{array}{c}\text { Exatidão } \\
\text { inter-dia } \\
\text { (três dias } \\
\text { consecutivos) }\end{array}$ & $\begin{array}{c}\text { Exatidão } \\
\text { intra-dia }\end{array}$ \\
\hline 2,5 & 8183886 & 26104 & 3,2 & 1,3 & 102,87 & 100,30 \\
1,25 & 367053 & 13287 & 3,6 & & & 100,51 \\
0,625 & 180395 & 4769 & 2,6 & 1,0 & 98,59 & \\
0,313 & 72341 & 5506 & 7,6 & & & 121,42 \\
0,156 & 36630 & 7488 & 20 & 18 & 130,59 \\
\hline
\end{tabular}

*média de no mínimo 6 injeções; CV - coeficiente de variação

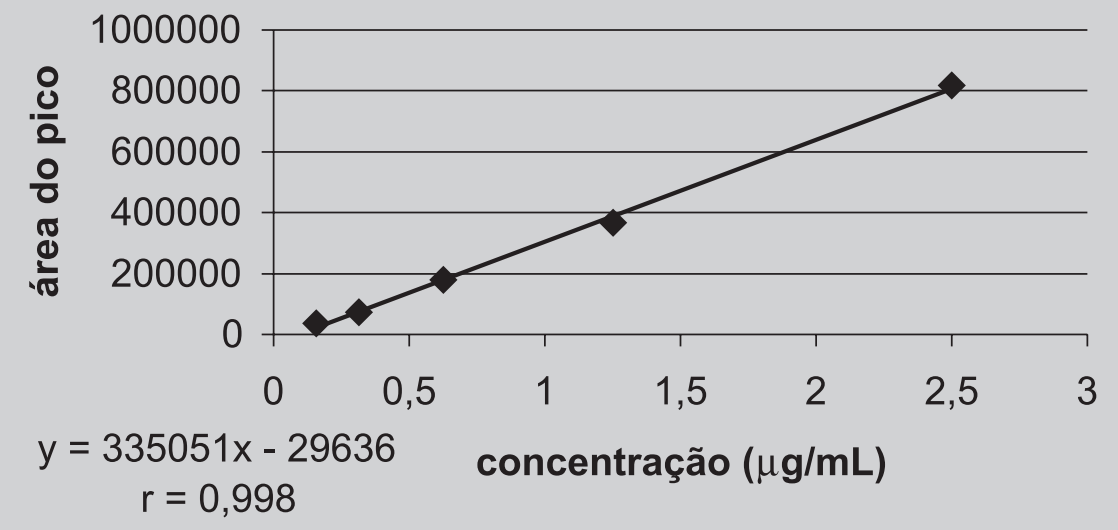

FIGURA 3 - Curva de calibração do 4-NC no intervalo de concentração de 0,156 a 2,5 $\mu \mathrm{g} / \mathrm{mL}$ de 4-NC dissolvido em etanol, referente à linearidade do método. 
TABELA III - Dados de recuperação de 4-NC adicionado ao homogeneizado de pele

\begin{tabular}{cccc}
\hline Concentração $(\mu \mathrm{g} / \mathrm{mL})$ & Área do pico * & Desvio padrão & Recuperação (\%) \\
\hline 2,5 & 8487395 & 85824 & 103,7 \\
0,625 & 165573 & 20920 & 91,7 \\
0,156 & 33609 & 8587 & 91,7 \\
\hline
\end{tabular}

*média de, no mínimo, três extrações

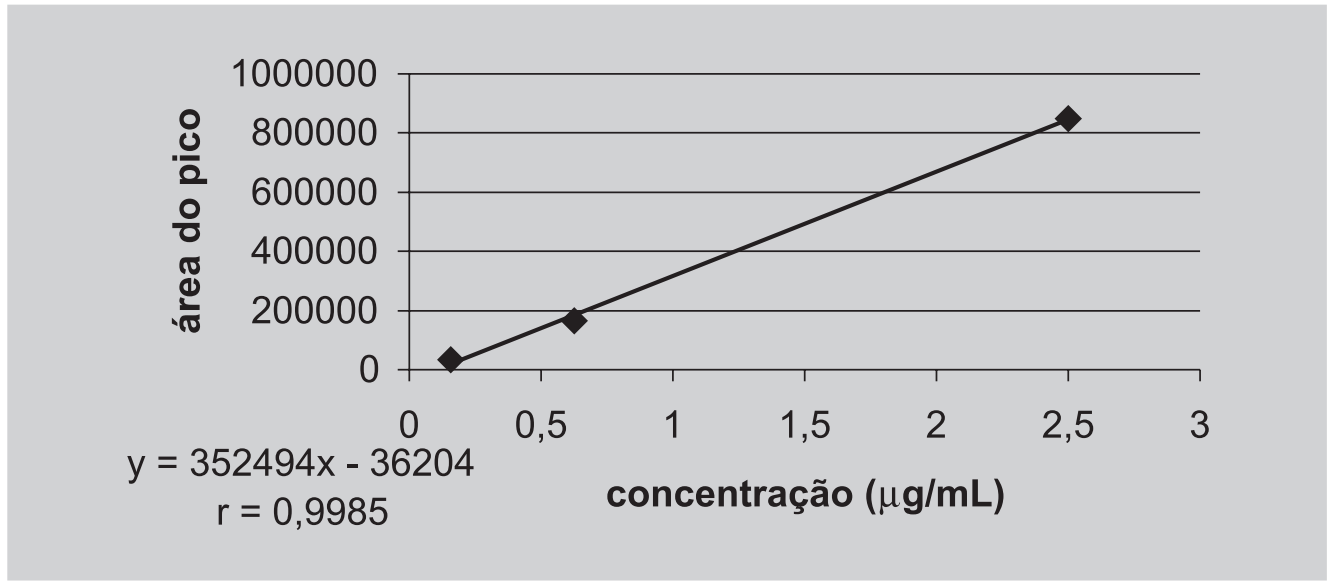

FIGURA 4 - Cromatogramas do padrão de 4-NC linear da área do pico do padrão de 4-nerolidilcatecol em função da concentração, para o 4-nerolidilcatecol recuperado do homogeneizado de pele.

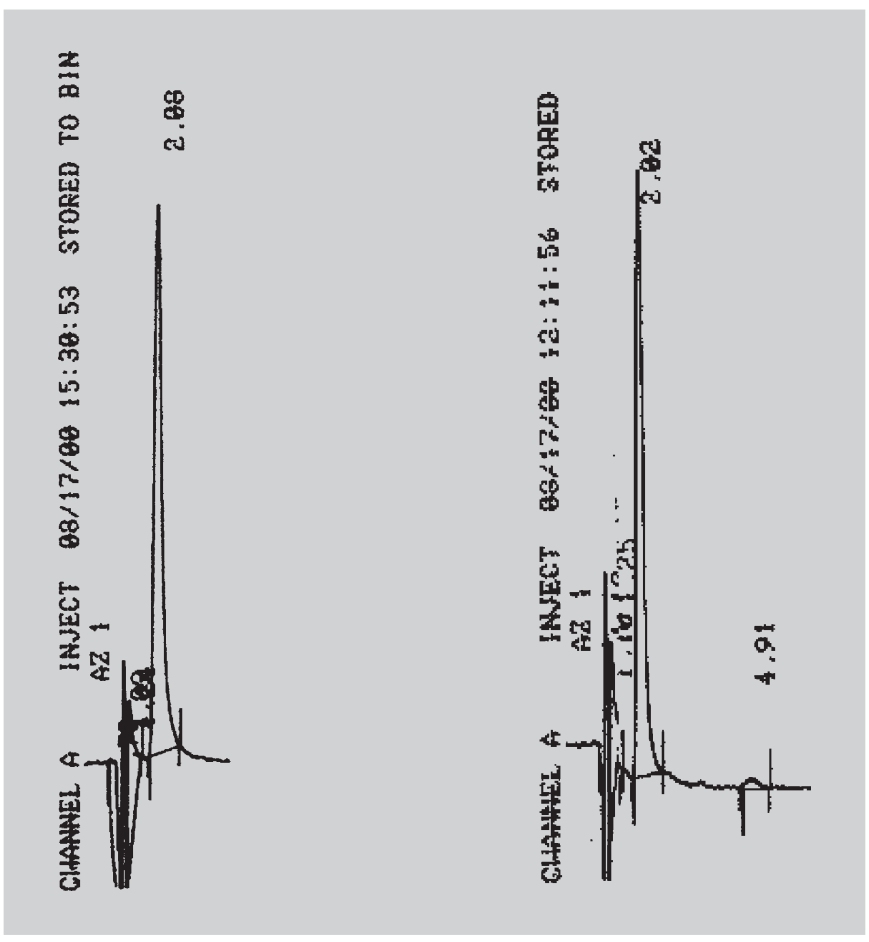

FIGURA 5 - Cromatogramas do padrão de 4-NC na concentração de $2,5 \mu \mathrm{g} / \mathrm{mL}$ em etanol (A) e cromatograma do 4-NC recuperado do homogeneizado de pele na concentração de $2,5 \mu \mathrm{g} / \mathrm{mL}$ (B).

\section{Ensaio de estabilidade do 4-NC no meio receptor do ensaio de permeação}

O 4-NC se mostrou estável durante o período do experimento, como foi comprovado pela análise estatística.

\section{Validação do método analítico para $\alpha$-tocoferol}

A precisão e estabilidade (intra e interdia) foram avaliadas empregando o $\alpha$-tocoferol nas concentrações de 0,5; 0,1 e 0,025 $\mu \mathrm{g} / \mathrm{mL}$ em etanol (Tabela 6). O limite de detecção foi de $0,015 \mu \mathrm{g} / \mathrm{mL}$ e o limite de quantificação foi de $0,045 \mu \mathrm{g} / \mathrm{mL}$ para a avaliação do o $\alpha$-tocoferol dissolvido em etanol. A figura 6 mostra a relação linear entre as concentrações do $\alpha$-tocoferol e a área dos picos, obtendo-se o coeficiente de correlação de 0,999 .

\section{DISCUSSÃO}

A perda de antioxidantes naturais presentes na pele, como resultado da irradiação ultravioleta, pode desencadear danos oxidativos, sob a influência de espécies reativas de oxigênio e nitrogênio (Podhaisky et al., 2002). A prevenção deste dano oxidativo tem sido objeto de inú- 
TABELA IV - Dados da curva de calibração e avaliação da precisão e exatidão da análise do $\alpha$-tocoferol

\begin{tabular}{lcccccc}
\hline $\begin{array}{c}\text { Concentração } \\
(\mu \mathrm{g} / \mathrm{mL})\end{array}$ & $\begin{array}{c}\text { Média da } \\
\text { área do pico* }\end{array}$ & $\begin{array}{c}\text { Desvio } \\
\text { Padrão }\end{array}$ & $\begin{array}{c}\text { CV\% inter- } \\
\text { corrida }\end{array}$ & $\begin{array}{c}\text { CV\% intra- } \\
\text { corrida }\end{array}$ & $\begin{array}{c}\text { Exatidão } \\
\text { intra-dia }\end{array}$ & $\begin{array}{c}\text { Exatidão } \\
\text { inter-dia }\end{array}$ \\
\hline 0,5 & 644243,3 & 21855 & 3,4 & 3,8 & 100,63 & 101,44 \\
0,25 & 307003,6 & 8585 & & & & 100,96 \\
0,1 & 104814,1 & 3825 & 3,6 & 3,4 & & 102,889 \\
0,05 & 53074,16 & 2416 & & & 134,29 & 129,09 \\
0,025 & 26666,0 & 2779 & 10,4 & 10,35 & & \\
\hline
\end{tabular}

*média de, no mínimo, dez injeções

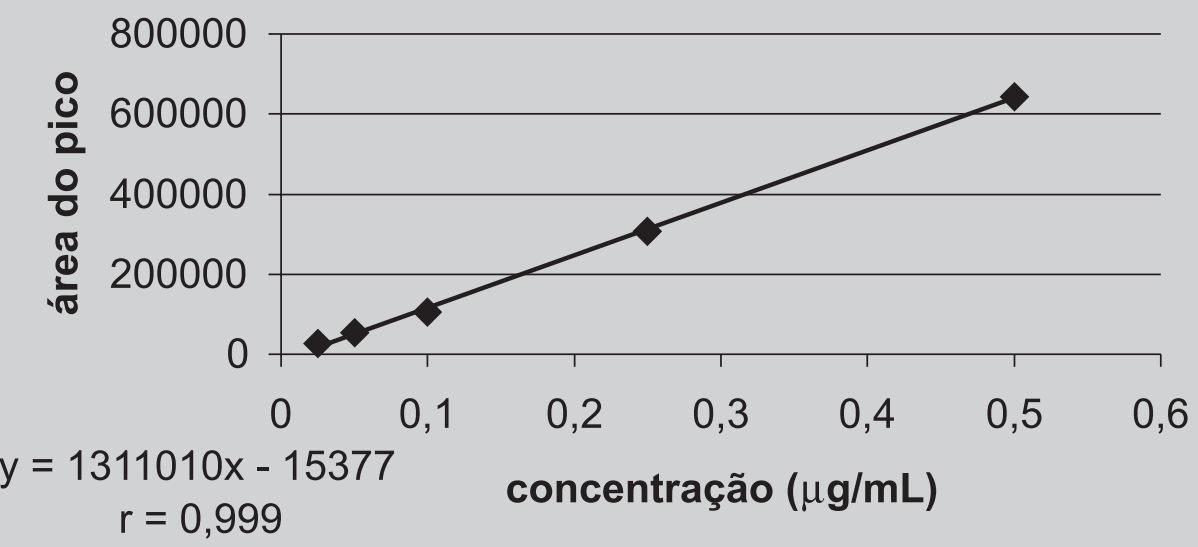

FIGURA 6 - Curva de calibração do $\alpha$-tocoferol em etanol no intervalo de concentração de 0,025 a $0,5 \mu \mathrm{g} / \mathrm{mL}$, referente à linearidade do método.

TABELA V - Dados de recuperação de $\alpha$-tocoferol adicionado ao homogeneizado de pele

\begin{tabular}{cccc}
\hline Concentração $(\mu \mathrm{g} / \mathrm{mL})$ & Área do pico * & Desvio padrão & Porcentagem de recuperação $(\%)$ \\
\hline 0,5 & 605788 & 51178 & 94,03 \\
0,1 & 116555 & 6941 & 111,20 \\
0,025 & 18800 & 985 & 80,7 \\
\hline
\end{tabular}

*média de, no mínimo, três extrações

meras investigações, sendo uma das metas o desenvolvimento de métodos farmacológicos e dietéticos para prevenção ou atenuação deste processo oxidativo (LopezTorres et al., 1998; Savouré et al., 1996). Neste contexto, o desenvolvimento de métodos analíticos validados, com aplicação em estudos de atividade antioxidante de princípios ativos que tenham potencial de combater o estresse oxidativo cutâneo é de grande relevância.

Os procedimentos para extração do $\alpha$-tocoferol e do 4-nerolidilcatecol foram considerados simples de custo 


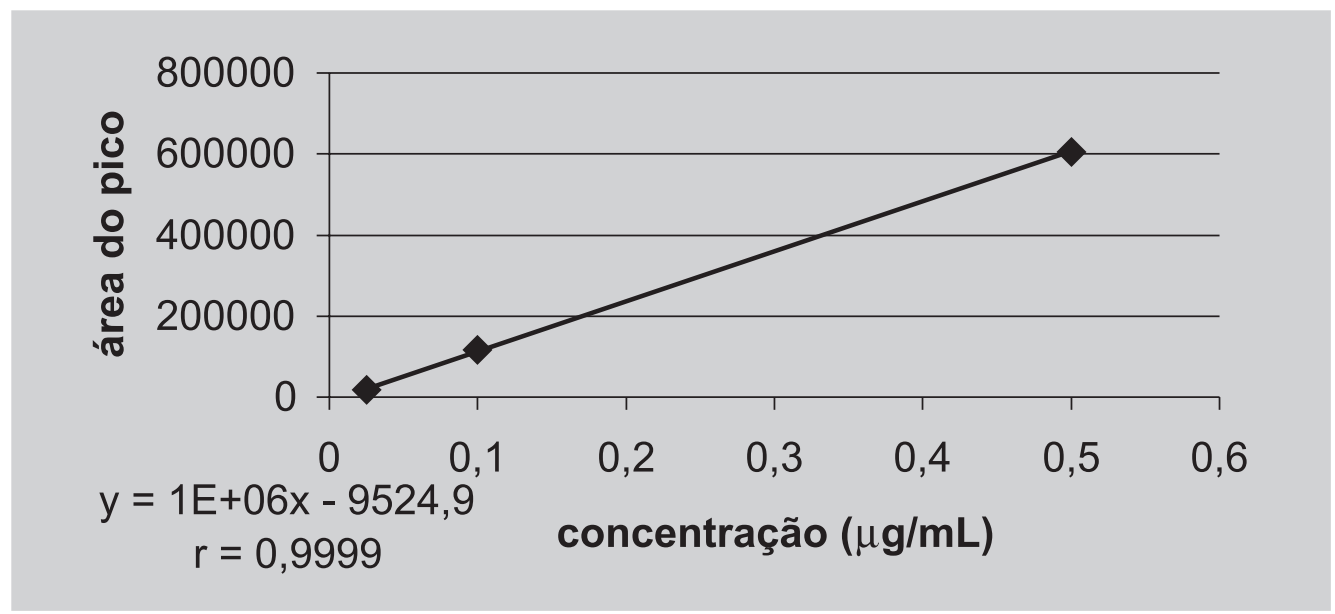

FIGURA 7 - Curva de calibração do $\alpha$-tocoferol recuperado do homogeneizado de pele.

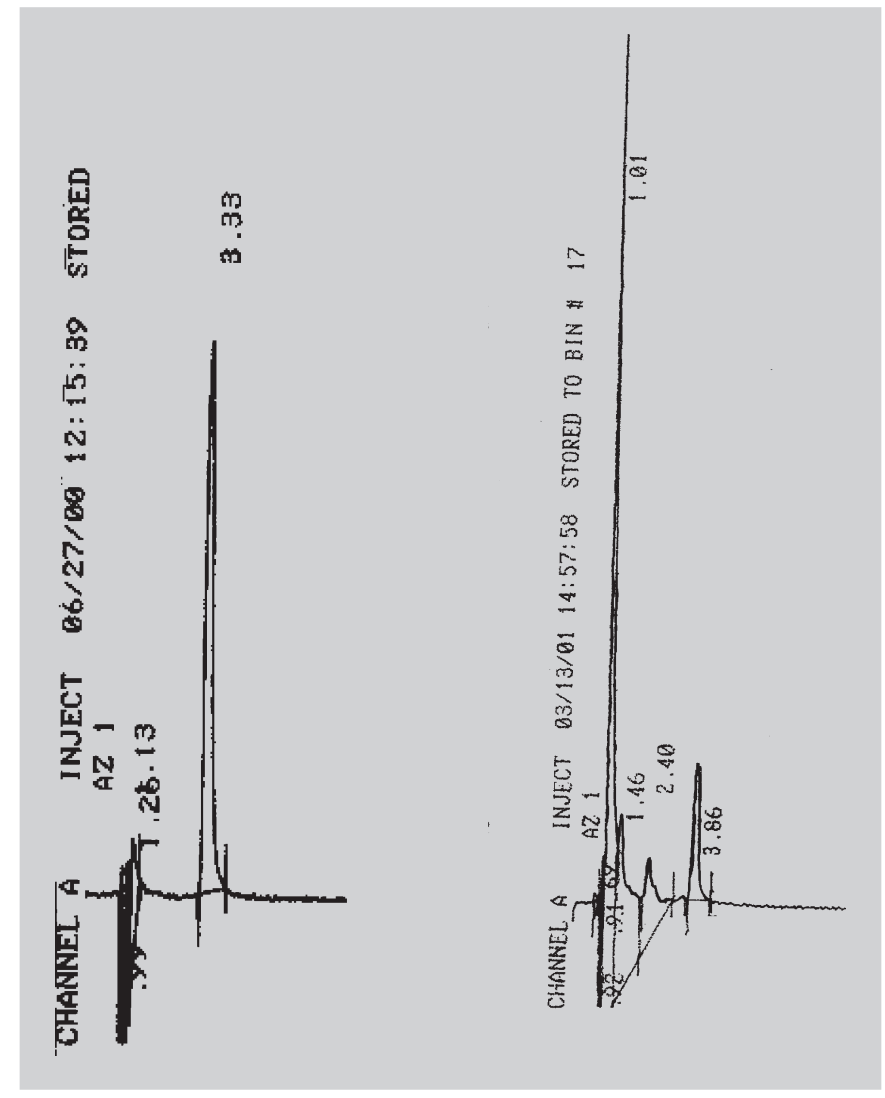

FIGURA 8 - Cromatogramas do padrão de $\alpha$-tocoferol na concentração de $0,5 \mu \mathrm{g} / \mathrm{mL}$ em etanol (A) e do $\alpha$-tocoferol extraído do homogeneizado de pele não adicionado (controle) (B). Tempo de retenção 3,33.

operacional e reduzido e com resultados satisfatórios para os objetivos propostos.
Comparando os parâmetros de validação, como precisão e exatidão, observamos que o solvente etanol:água conferiu maior confiabilidade à análise de concentrações mais baixas, do que quando foi utilizado o etanol puro para solubilizar o 4-NC. Isto provavelmente está relacionado à volatilidade do etanol que é maior que a da mistura de solventes, causando maior variabilidade dos resultados de avaliação das concentrações mais baixas. Apesar disto os limites de detecção e quantificação da curva em etanol:água se mostraram adequados para avaliação das amostras retiradas do compartimento receptor da célula de Franz, utilizada nos estudos de permeação do 4-NC (Ropke et al., 2002), assim como para análise de extratos hidroalcoólicos de Pothomorphe umbellata.

Também foi interessante observar a maior sensibilidade do método para $\alpha$-tocoferol do que para o 4-NC. A sensibilidade de um método analítico é determinada a partir da inclinação da reta de calibração. (Causon, 1997). No caso do $\alpha$-tocoferol esta inclinação foi aproximadamente três vezes maior (1.311.010 para 335051) do que a da reta de calibração do 4-nerolidilcatecol dissolvido em etanol. Isto significa que pequenas alterações na concentração de $\alpha$-tocoferol acarretarão variações maiores de resposta do detector.

Os resultados de recuperação e precisão inter e intradia, apresentados nas Tabelas I, II, III, IV e V, demonstram a aplicabilidade do procedimento analítico padronizado, no intervalo das concentrações analisadas (ANVISA, 2002). Portanto, conclui-se que a metodologia analítica validada é perfeitamente aplicável na avaliação da concentração de 4-NC e $\alpha$-tocoferol em homogeneizados de pele. 


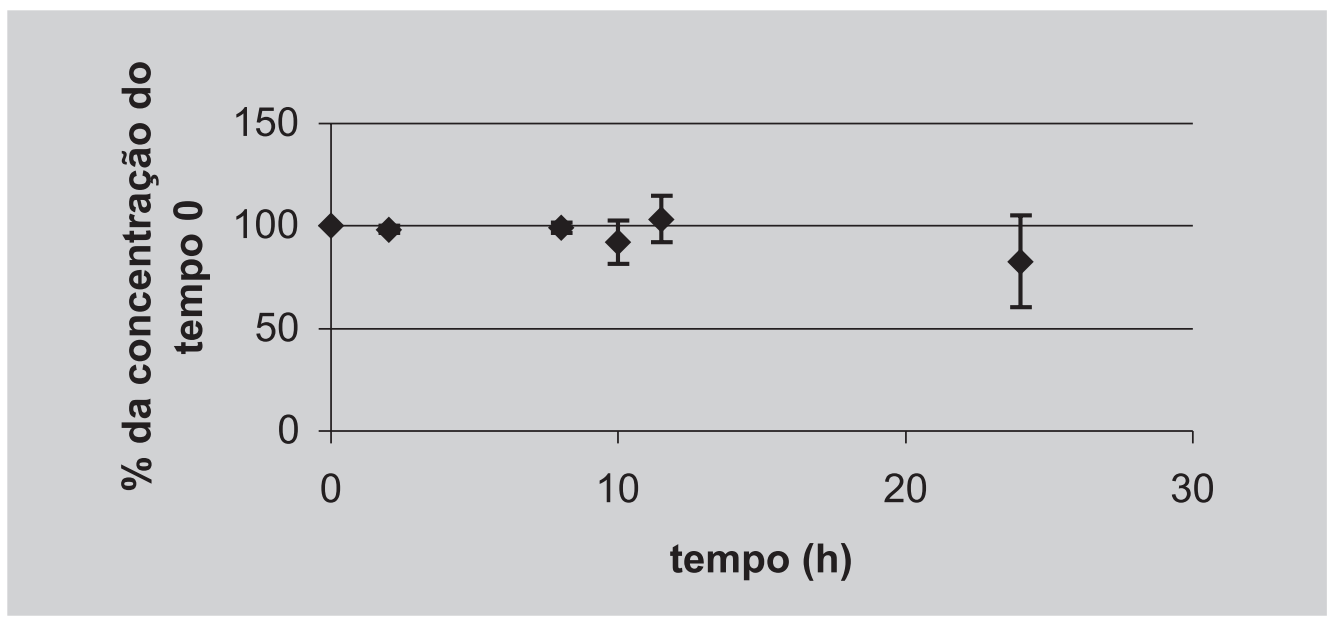

FIGURA 9 - Variação da concentração de 4-nerolidilcatecol numa solução etanol:água 1:1 à $37^{\circ} \mathrm{C}$ nos tempos $8,10,11,5$ e $24 \mathrm{~h}$, em relação ao tempo 0 . A variação não foi considerada estatísticamente significante (ANOVA, $\mathrm{F}=1,272$ e p = 0,3434).

\section{AGRADECIMENTOS}

À Fapesp, pela bolsa de doutorado concedida a Cristina Dislich Ropke. Elissa Arantes Ostrosky foi bolsista de mestrado CNPq, Renata Rodrigues Meirelles e César Moisés Camillo, bolsistas de Iniciação Científica $\mathrm{PIBIQ/CNPq.}$

\section{ABSTRACT}

\section{Validation of $\alpha$-tocopherol and 4-nerolidylcathecol quantitative assessment methodologies}

Topical administration of antioxidants, such as $\alpha$ tocopherol $(\alpha-T)$, provides an efficient manner of enriching the endogenous cutaneous protection system, and it constitutes a successful strategy for diminishing the ultraviolet radiation-mediated oxidative damage. Besides $\alpha$-tocopherol the use of other natural occurring compounds with antioxidant activity has been proposed for the same purpose. The aim of this study was to develop a validated analytical method for the determination of atocopherol and 4-nerolidylcathecol (4-NC) concentrations in skin homogenates in a pharmaceutical formulations. We employed liquid chromatography with electrochemical detection. Chromatography was performed on a Supelcosil LC-8, $3 \mathrm{~mm}, 75 \times 4.6 \mathrm{~mm}$ column (Supelco, Bellefonte, PA, USA) with a mobile phase of methanol:water (9:1) for 4-NC and (95:5) for aT, both containing $20 \mathrm{mM} \mathrm{LiClO} 4$ and $2 \mathrm{mM} \mathrm{KCl}$. The flow rate was set at $1.0 \mathrm{ml} / \mathrm{min}$. We established validation parameters including sensitivity, precision, accuracy, stability and found a linear relationship between the concentrations ranges of $0.025 \mu \mathrm{g} / \mathrm{mL}$ to $0.1 \mu \mathrm{g} / \mathrm{mL}$ of $\alpha$ Tand $0.15 \mathrm{mg} / \mathrm{mL}$ to $2.5 \mathrm{mg} / \mathrm{mL}$ of $4-N C$. The recovery of $\alpha$-T from skin homogenates was $94.03,111.2$ and $80.7 \%$ for the concentrations of $0.5,0.1$ and $0.025 \mu \mathrm{g} / \mathrm{mL}$ respectively. The recovery for the following concentrations of 4-NC: $2.5,0.625$ and $0.156 \mu \mathrm{g} / \mathrm{mL}$ was 103.7, 91.7 and $91.7 \%$. This analytical procedure has been successfully employed in cutaneous permeation studies, antioxidant activity studies and determinations of 4-NC in Pothomorphe umbellata root extracts.

UNITERMS: Pothomorphe umbellata. 4-nerolidylcathecol. HPLC. Validation

\section{REFERÊNCIAS BIBLIOGRÁFICAS}

BARROS, S. B. M.; TEIXEIRA, D. S.; AZNAR, A. E.; MOREIRAJÚNIOR.; J.A.; ISHII, I.; FREITAS, P. C. D. Antioxidant activity of ethanolic extracts of Pothomorphe umbellata L. Miq. Ciênc. Cult, São Paulo, v.48, p.114-116, 1996.

BISSET, D. L.; CHATERJEE, R.; HANNON, D. P. Photoprotective effect of superoxide-scavenging antioxidants against ultraviolet radiation induced chronic skin damage in hairless mouse. Photodermatol. Photoimmunol. Photomed., Copenhagen, v.7, p.56-62, 1990.

BLACK, H. S. Potential involvment of free radical reactions in ultraviolet light - mediated cutaneous damage. Photochem. Photobiol., Oxford, v.46, n.2, p.213-221, 1987. 
BONINA, F.; LANZA, M.; MONTENEGRO, L.; PUGLISI, C.; TOMAINO, A.; TROMBETTA, D.; CASTELLI, F., SAIJA, A. Flavonoids as potential protective agents against photo-oxidative skin damage. Int. J. Pharm., Amsterdam, v. 145, p. 87-94,1996.

BURTON, G. W.; WEBB, A.; INGOLD, K. U. A mild, rapid and efficient method of lipid extraction for use in determinig vitamin E/lipid ratios. Lipids, Champaign, v.20, p.29-39, 1985.

CAUSON, R. Validation of chromatographic methods in biomedical analysis. Viewpoint and discussion. $J$. Chromatography, Amsterdam, v.689, p.175-180, 1997.

FUCHS, J. Potential and limitations of the natural antioxidants RRR-alpha-tocopherol, L-ascorbic acid and b-carotene in cutaneous photoprotection. Free Radical Biol. Med., New York, v. 25, p. 848-873, 1998.

GUIA PARA VALIDAÇÃO DE MÉTODOS ANALÍTICOS - publicado pela ANVISA através da resolução - RE n 475, de 19 de março de 2002. Disponível em: www.anvisa.gov.br/hotsite/genericos/ legis/resolucoes/2002/475_02re.htm. Acesso em 28 jul. 2002.

JUERKIEWICZ, B. A.; BUETTNER, G. R. EPR detection of free radicals in UV-irradiated skin: mouse versus human. Photochem. Photobiol., Oxford, v.64, p.918-922, 1996.

KIJJOA, A.; GIESBRECHT, A. M.; AKISUE, M. K.; GOTTlieB, O. R.; GOTTlieB, H. E. 4Nerolidylcathecol from Pothomorphe umbellata. Planta Med., Stuttgard, v.39, p.85-87, 1980.

LOPEZ-TORRES, M.; THIELE, J. J.; SHINDO, Y.; PACKER, L. Topical application of $\alpha$-tocopherol modulates the antioxidant network and diminishes ultraviolet-induced oxidative damage in murine skin. British J. Dermatol., Oxford, v.138, p.207-215, 1998.
MAYER, P. The effect of vitamin E on the skin. Cosmet. Toiletries, Oak Park, v.108, p.99-109, 1993.

PODHAISKY, H. P.; RIEMSCHNEIDER, S.; WOHLRAB, W. UV light and oxidative damage of the skin. Pharmazie, v.57, p.30-33, 2002.

ROPKE,C. D; KANEKO, T. M.;RODRIGUES, R. M.; SILVA,V. V.; BARROS, S.; SAWADA, T. C. H.; KATO, M. J.; BARROS, S. B. M. Evaluation of percutaneous absorption of 4-nerolidylcathecol from four topical formulations. Int. J. Pharm., Amsterdam, v.249, p.107114, 2002.

ROPKE, C. D. Avaliação da atividade antioxidante de Pothomorphe umbellata na pele. CONGRESSO BRASILEIRO DE COSMETOLOGIA DA ABC, 14 ., São Paulo, 2000. Anais. São Paulo: Tec Art Editora, 2000. $700 \mathrm{p}$.

SAIJA, A.; TOMAINO, A.; TROMBETTA, D.; DE PASQUALE, A.; UCCELLA, N.; BARBUZZI, T.; PAOLINO, D.; BONINA, F. In vitro and in vivo evaluation of caffeic and ferulic acids as topical photoprotective agents. Int. J. Pharm., Amsterdam, v.199, p. 39-47, 2000.

THIELE J. J.; TRABER, M. G.; PACKER, L. Depletion of human stratum corneum vitamin E: an early and sensitive in vivo marker of UV-induced photo-oxidation. J. Invest. Dermatol., Baltimore, v.110, p.756-761, 1998.

UNITED States pharmacopeia. 23. ed. Rockville: United States Pharmacopeial Convention, 1995. p.864-865.

Recebido para publicação em 13 de agosto de 2002. 